\title{
ON THE IMPROVEMENT OF CALIBRATION ACCURACY OF PARALLEL ROBOTS - MODELING AND OPTIMIZATION
}

\author{
Toufik Bentaleb \\ Control Department, LAMIH UMR CNRS 8201 (Laboratory of Industrial and Human Automation Control, \\ Mechanical Engineering and Computer Science), University of Valenciennes, Valenciennes, France \\ e-mail: bentaleb.toufik@gmail.com \\ JAMSHED IQBAL \\ University of Jeddah, College of Engineering, Department of Electrical and Electronic Engineering, Jeddah, Saudi Arabia \\ Department of Electrical Engineering, FAST National University, Islamabad, Pakistan \\ e-mail: jmiqbal@uj.edu.sa
}

\begin{abstract}
This paper proposes kinematic based calibration methods for Delta parallel robots. The boundary of the robot workspace is computed using a forward kinematic model. Influence of errors in kinematic parameters on the workspace boundaries is investigated. The novelty of the proposed approach lies in Jacobian-based computation of kinematic models. Also, the present work extends and applies the existing calibration methods traditionally meant for serial robots on the Delta robot. These methods include the forward method and the inverse method. Simulation results confirm the efficacy of the proposed calibration strategies.
\end{abstract}

Keywords: robot calibration, error analysis, parallel robots, kinematic, modelling

\section{Introduction}

For some industrial applications such as High Speed Machining (HSM) or fast handling, serial kinematics manipulators appear to be best suited (Alam et al., 2018). Indeed, this type of architecture implies that each motor axis supports its successive link (Iqbal et al., 2016; Bentaleb and Shahin, 2015). However, this results in degradation of the dynamic performance due to accumulation of the masses in motion. Consequently, parallel robots attract a great attention in robotics and mechatronics community owing to their distinguishing features compared with other robot types such as serial robots (Ajwad et al., 2018). Superior accuracy in object manipulation, high compactness and rigidity in parallel robots lead to vast dynamics capabilities, thus making them a best choice in several application areas. Nowadays, parallel kinematic manipulators are common in medical, space, industrial and other HSM applications requiring high positional accuracy. However, it is necessary to improve its repeatability and positional accuracy, which is a big challenge because of calibration issues. Generally, parallel robots require calibration to improve the precision performance during task execution. The accuracy synthesis and kinematic calibration can be used to solve this problem. Theoretically, for a six degree of freedom (DOF) parallel mechanism, the error can be compensated by kinematic calibration. However, for mechanisms having less DOF than six, the accuracy synthesis is important because they have uncompensable end errors as pointed in (Mei et al., 2009). A three-DOF parallel manipulator named 'Delta' belongs to this kind of mechanism.

Literature reports several techniques to address the calibration problem of Delta robots. Bai et al. (2015) used distance measurements from a laser tracker to calibrate Delta robot. Based on the derived kinematic model of error, they classified the source of errors affecting pose accuracy. The set of acquired tracker data was used to fully or partially identify the source of error. To 
compensate error in real-time, a linearized actuator was designed and developed. Considering a cylindrical work-envelope, results dictated that error in uncompensated positional accuracy was suppressed so as to improve the kinematic calibration. In contrast to Bai's work, the calibration approach presented by Fan et al. (2015) for parallel mechanisms does not rely on measurement of posture/position. Instead, they used forward kinematics of the robot in conjunction with genetic algorithm that identified kinematic parameters by solving the discrete optimization problem. The proposed calibration approach improved accuracy by a factor of $78.3 \%$. Given the fact that genetic algorithm does not result in a unique optimal solution, it was recommended to execute the calibration procedure multiple times prior to selection of the best result.

A recent development in this subject is elasto-geometrical calibration proposed in (Kamali et al., 2016). This approach used end-effector positional data acquired by a laser tracker to identify parameters related with joint stiffness and errors. This work considered a wide range of torques and forces to measure position errors for each configuration of the robot. The observability analysis reduced the sensitivity of calibration accuracy w.r.t. unmodelled errors and noise. The results of various configurations subjected to external loads dictated that the over-performance of the presented approach compared to the customary kinematics-based calibration strategy. Another novel approach to enhance robot accuracy is reported in (Nguyen et al., 2015) which integrates Extended Kalman Filter (EKF) based algorithm and Artificial Neural Network (ANN). EKF was used to model and identify geometric parameters while ANN compensated non-geometric un-modelled sources of error. Simulation results on serial PUMA manipulator confirmed the correctness of the proposed strategy and proved its over-performance compared to the conventional approaches relying on joint compliance parameters and link geometry.

The absolute calibration approach proposed by Nubiola and Bonev (2014) was based on a 6D measurement system, which mainly comprises a COTS telescoping ballbar and two custom developed fixtures. Considering a small six-axis serial robot, a laser tracker was used to validate the calibration strategy in 10000 random robot configurations. An improvement in mean positioning error from 0.873 to 0.479 was reported. Pagis et al. (2015) presented a promising robust approach to enlarge work-envelope of parallel robots. The defined optimal trajectory permits crossing Type-2 singularities. The generated trajectory mitigated the degeneration of the system dynamics in the vicinity of the singularity locus. The multi-mode control law then tracked this trajectory. A five-bar planar mechanism was used to validate the developed control laws. Zhao et al. (2015) introduced a novel medical robot with a parallel structure intended for diagnosis of vessel disease in lower limbs of humans. With a rigorous analysis of robot accuracy and repeatability, the work also reported a calibration procedure based on the identification approach to identify kinematic parameters. Being a simple and easily realizable method, the proposed method does not require advance knowledge of calibration approaches. The experimental results demonstrated that the calibration process can improve the position error from $6 \mathrm{~mm}$ to less than $1 \mathrm{~mm}$.

The key objective behind the kinematic calibration of robots is to obtain the values of kinematic parameters. Removing uncertainty about these settings allows adjustment of the mathematical models or virtual models to more accurately position the robots. In fact, in most cases, the robot positioning errors are due to poor fitting of the parametric values as highlighted in (Mo et al., 2016; Axehill et al., 20141). Conventional methods of calibration of parallel robots, such as those reported in (Yu, 2008; Yu et al., 2005; Rauf and Ryu, 2001; Besnard and Khali, 2001) are expensive in terms of time and computational cost because of 3D measurement devices, and also suffer from other constraints due to inaccuracy of measurement systems and installation complexity (Meng and Zhuang, 2007). Other notable methods are based on semi-autonomous calibration procedures employing additional sensors mounted only on passive joints of the robot instead of an external sensor. The proposed method in the present paper extends Zhuang's approach for Delta parallel robot. 


\section{Description of Delta robot}

Delta parallel robot was developed by Reymond Clavel in 1985 (Viera et al., 2011). Distinguishing features of this robot include: high work rate, superior accuracy and less power consumption. The coordinate points needed for the modeling of this structure are: $A_{i}$ - motorized joints center between the arms and the base, $i=1,2,3, B_{i}$ - passive joint between the two arms, $C_{i}$ - passive joint between the arm and to its connected mobile platform, $O_{0}$ - center of the fixed platform and $O$ - center of the mobile platform. The point $O_{0}$ is the origin of the coordinate system $R_{0}$ associated with the base, and $x_{0}$ axis passes through the points $A_{i} . z_{0}$ axis and the normal of the planar surface is denoted by $A_{i}$. The dimensioned parameters of the model are: $L a_{i}=260 \mathrm{~mm}$, $L b_{i}=480 \mathrm{~mm}$ of the arm $i$ and the parameter $R$ is the difference between radii of the base and the mobile platform $\left(R b=194 \mathrm{~mm}\right.$ and $\left.R_{n}=30 \mathrm{~mm}\right)$. The angular parameters are: the joint variable $\alpha_{i}$ of each arm $i$ and the angle $\theta_{i}\left(0^{\circ}, 120^{\circ}\right.$ and $\left.240^{\circ}\right)$ which corresponds to the orientation of axes $z_{i}$ of each joint actuated revolution point $A_{i}$. The cornerstone of the mobile structure is the parallelogram consisting of three kinematics chains that connects the static platform to the moving platform. Regardless of its movements and position, the three orientations of the moving platform are kept constant without any influence of the motor. The moving platform is the body terminal of the robot with 3-DOF. The structure has a ternary symmetry consisting of three identical kinematic chains arranged in $120^{\circ}$. The lengths and angles parametric characteristics of this structure are shown in Fig. 1.

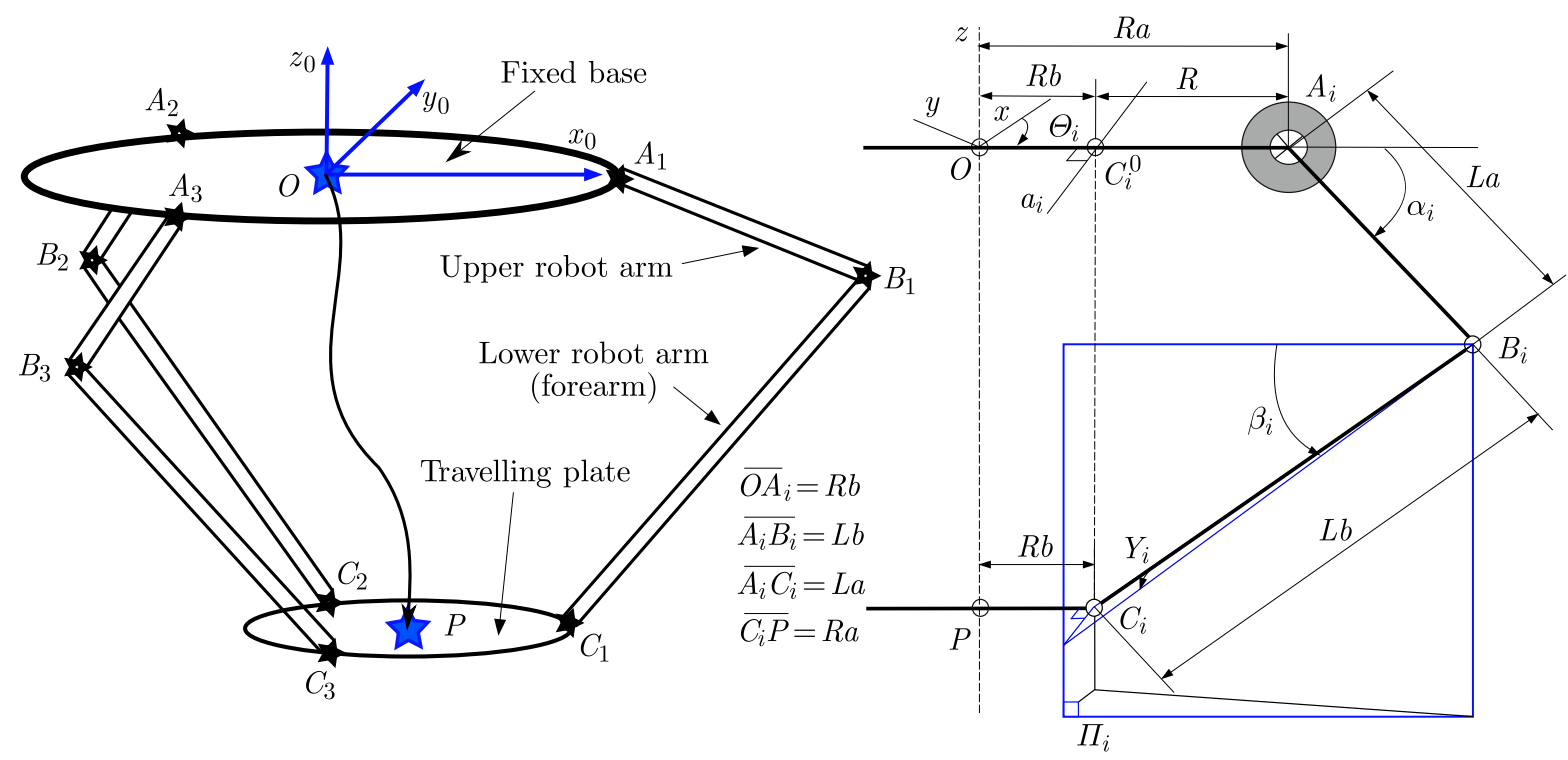

Fig. 1. Left: schematic representations of Delta robot with the definition of the coordinate system by placing the origin at the fixed base (base-plate). Right: lengths and angles parametric characteristics of Delta robot. Each parallelogram is represented by a single segment

The nomenclature is as follows: $R a$ is the distance between the center of the static platform and the axis of rotation of the arm, $R b$ is the distance between the center of the moving platform and the side of the parallelogram associated with the moving platform, $L a$ is the arm length, $L b$ is length of the parallel bar, $R$ is the difference between $R a$ and $R b$. This distance defines the position of the point $C_{i}$ on the line $a_{i}$ which is perpendicular to the line $O A_{i}$. Angle characteristics of this structure are: $\alpha_{i}$ is the angle between the $i$-th arm and the plane of the static platform, by convention, this angle is positive when the arm is located on the side of the mobile platform, $\beta_{i}$ is the angle between the plane of the $i$-th parallelogram and the horizontal plane measured in a vertical plane $\pi_{i}$ containing the arm $i, \gamma_{i}$ is the angle between the vertical plane $\pi_{i}$ and bars of the parallelogram $i, \theta_{i}$ is the angle between the plane $\pi_{i}$ and the plane $O x z$. The three orientations 
of the robot end-effector are constant. The kinematics can simplify the robot geometry. This simplification is obtained by translation of the whole-arm parallel bars, thereby combining the points $O_{i}$ and $A_{i}$. The same simplification concept is applied to points $B_{i}$ and $P$. In our previous work (Bentaleb et al., 2007), the kinematic modeling of robots with closed structures was based on an open loop kinematic model considering each chain individually according to the constraints imposed by other chains. The derived models serve as basis to identify the constraints necessary for calibration and to analyze workspace and accuracy.

\section{Calibration methods}

Two kinematic calibration approaches which are traditionally used to calibrate serial robots are presented in this Section. The first approach is based on Forward Kinematic Model (FKM) of Delta robot while the second strategy is based on Inverse Kinematic Model (IKM) of the robot. These two kinematic approaches have been tested and applied to another type of parallel robots "Gough platform" (Merlet, 2006). The two approaches are detailed below.

\subsection{FKM-based method}

The control of actuators allows the robot to reach a certain configuration $X$. This configuration is computed using FKM. So, we can obtain $X^{F K M}=F_{F K M}(P, \alpha)$ based on values of the actuators $\alpha$ and geometric parameters $P$. The exact position of the manipulator $X^{r}$ is calculated and then compared with the result given by FKM to determine the position error of the manipulator, which is related with unknown geometrical parameters i.e.

$$
\partial X=X^{r}-X^{f k m}(P, \alpha)
$$

In this method, the main concept behind calibration is to deal with the geometric parameters to decrease the position error as much as possible.

\subsection{IKM-based method}

Instead of evaluating the position error in the operational space, the IKM-based method relies on indirect quantification of the error on the joint values in the coordinate space. For a known reference position of the manipulator $X^{r}$, the joint coordinates are calculated using IKM based on geometric parameters $P^{i}$ of the robot. Then $\alpha_{i}^{i k m}$ of each segment is obtained using

$$
\alpha_{i}^{i k m}=F_{i k m}\left(P_{i}, X^{r}\right)
$$

These information values are compared with the actual values of the joint coordinates $\alpha^{r}$ i.e.

$$
\Delta \alpha_{i}=\alpha_{i}^{r}-\alpha_{i}^{i k m}\left(P_{i}, X^{r}\right)
$$

The goal is that for each arm segment $i$, the geometric parameters are modified to decrease $\Delta \alpha$ error as much as possible. The main advantage of this method is that the error for a given segment is independent of errors in other segments of the robot. Calibration can be decomposed into successive identification of the parameters $P^{i}$ of each segment. Thus, the number of unknowns can be determined by the number of manipulator segments ( 3 for Delta robot) consequently resolving the problem. 


\subsection{Solution of equations}

\subsubsection{Using FKM}

The position error $\varepsilon_{k}^{f k m}$ for a configuration of the manipulator $k$ gives the constraint as

$$
\varepsilon_{k}^{f k m}(P)=X_{k}^{f k m}-X^{f k m}\left(P, \alpha_{k}^{r}\right)
$$

Such that, for the exact parameters $P^{r}$ of the manipulator, the error is zero. The calibration problem leads to determining $P^{r}$ as follows

$$
\varepsilon_{k}^{f k m}\left(P^{r}\right)=X_{k}^{f k m}-X^{f k m}\left(P^{r}, \alpha_{k}^{r}\right)=0
$$

The solution of this problem requires having as many algebraically independent equations as the number of unknowns. Depending on the nature of the reference variables, the error $\varepsilon_{k}^{f k m}$ provides $N_{m}$ equations where sensory instrumentation gives the position (3 parameters) of the mobile platform. The vector $X_{k}^{r}$ provides three independent information values, i.e. $N_{m}=3$. If we place the mobile plate in $N_{c}$ reference configurations, we get $N_{c}$ types of relationships and thus $N_{m} \times N_{c}$ equations. The number $N_{p}$ of the geometrical parameters to be identified is dependent on the selected robot model. To determine $P$, as $\varepsilon_{k}^{f k m}(P)=0$, with $k=1, \ldots, N_{c}$, the inequality $N_{m} \times N_{c} \geqslant N_{p}$ holds true. Taking the case of calibration of Model 24, where the number 24 represents the number of parameters, i.e. $N_{p}=24$. So, for each segment, there are 8 parameters $(i=1-3)$ i.e.

$$
P_{i}=\left[D x_{i}, D y_{i}, D z_{i}, \theta_{i}, \alpha_{i}, L a_{x_{i}}, L a_{y_{i}}, L b_{i}\right]
$$

The minimum number of measured positions of the manipulator configuration is equal to $24 / 3=8$. The problem is to determine $P^{c}$, i.e. all parameters obtained by the calibration, such as

$$
\begin{aligned}
& \varepsilon_{1}^{f k m}\left(P^{c}\right)=\chi_{1}^{f k m}\left(P^{c}, \alpha_{1}^{r}\right)=0 \\
& \vdots \\
& \varepsilon_{k}^{f k m}\left(P^{c}\right)=\chi_{k}^{f k m}\left(P^{c}, \alpha_{k}^{r}\right)=0 \\
& \vdots \\
& \varepsilon_{N_{c}}^{f k m}\left(P^{c}\right)=\chi_{N_{c}}^{f k m}\left(P^{c}, \alpha_{N_{c}}^{r}\right)=0
\end{aligned}
$$

where $k=1, \ldots, N_{c}$ is the index of the robot configuration. The required parameters need to be determined for all the configurations corresponding to the known reference positions $\chi_{k}^{r}$ of the robot and for all angles $\theta_{k}^{r}$ of the mobile joints.

\subsubsection{Using $I K M$}

Instead of using the error in the angles of motorized joints and substitute in (3.3), we prefer to use a difference of square of the angles to simplify the IKM equations of IKM. There are several models of Delta robots depending on the choice of number of parameters (see Ibrahim and Khalil, 2010). For the position $k$ and exactly known manipulator configurations $\chi_{k}^{r}$ as well as the movable hinge angle $i$ to be calibrated, the IKM equation gives

$$
F_{i k m}^{i, k}\left(P_{i}, \chi_{k}^{r}, \alpha_{i, k}^{r}\right)=\left(\overline{C B}_{i, k}^{r}\right)^{\mathrm{T}} \overline{C B}_{i, k}^{r}-L b_{i}
$$

With this constraint equation, for $P_{i}=P_{i}^{r}$, we therefore have

$$
\varepsilon_{i, k}^{i k m}\left(P_{i}, \chi_{k}^{r}, \alpha_{i, k}^{r}\right)=F_{i k m}^{i, k}\left(P_{i}, \chi_{k}^{r}, \alpha_{i, k}^{r}\right)=0
$$


In order to obtain a finite set of solutions, we need as many algebraically independent equations as unknowns. However, (3.9) provides us a single $\left(N_{m}=1\right)$ equation for each segment $i$. For a configuration $k=1, \ldots, N_{c}$, there are $N_{c}$ constraint equations corresponding to $N_{c}$ reference positions. To identify all the $N_{p}$ geometric parameters $P_{i}$ for each segment $i$, it will require $N_{c} \geqslant N_{p}$. Thus, for Model 24, we have $N_{p}=8$ reference configurations. Since the constraint equations are defined independently for each segment, the same calibration data of all the other parameters of the robot can be used. To determine 24 geometric parameters, it will take at least 8 measurement configurations. The problem therefore is to determine $P_{i}^{c}$ such as

$$
\begin{aligned}
& \varepsilon_{i, 1}^{i k m}\left(P_{i}^{c}, \chi_{1}^{r}, \alpha_{i, 1}^{r}\right)=0 \\
& \vdots \\
& \left.\varepsilon_{i, k}^{i k m}\left(P_{i}^{c}, \chi_{k}^{r}, \alpha_{i, k}^{r}\right)\right)=0 \\
& \vdots \\
& \left.\varepsilon_{i, N_{c}}^{i k m}\left(P_{i}^{c}, \chi_{N_{c}}^{r}, \alpha_{i, N_{c}}^{r}\right)\right)=0
\end{aligned}
$$

\subsection{Solution}

The main challenge in calibration is that we do not know the exact placement $\chi_{r}^{r}$ and associated angles of the movable joints associated $\alpha_{r}^{r}$. An approximation of their values can be obtained by means of measuring devices. Therefore

$$
\chi_{k}^{r}=\chi_{k}^{m}+\varepsilon_{p}
$$

where $\chi_{k}^{m}$ is the measured position of the configuration $k$ and $\varepsilon_{p}$ is the error in the position induced by the measurement noise. Similarly,

$$
\alpha_{k}^{r}=\alpha_{k}^{m}+\varepsilon_{\theta}
$$

where $\alpha_{k}^{m}$ is the angle measured from the articulated joint $i$ of the configuration $k$ and $\varepsilon_{\theta}$ is the error in the angle of the movable joint $i$ induced by the measurement noise.

The idea is to determine $P=P^{r}$ in order to have $(3.13)_{1}$ for FKM and $(3.13)_{2}$ for IKM based methods for each segment $i$

$$
\begin{aligned}
& \min _{P=P^{c}} \sum_{k=1}^{N_{c}}\left\|\varepsilon_{k}^{F K M}(\chi_{k}^{m}, \alpha_{k}^{m}, \overbrace{P}^{\text {unknown }})\right\|^{2} \\
& \min _{P_{i}=P_{i}^{c}} \sum_{k=1}^{N_{c}}\left\|\varepsilon_{i, k}^{I K M}(\chi_{k}^{m}, \alpha_{i, k}^{m}, \overbrace{P_{i}}^{\text {unknown }})\right\|^{2}
\end{aligned}
$$

An optimization method offers several advantages. In fact, the measurement noise modifies the constraints of equation coefficients and, therefore, the solution does not lead to the exact root. Finding a solution that minimizes the criterion $c=\varepsilon^{\mathrm{T}} \varepsilon$ in $P$ better describes the calibration problem. The noise on the measurements and numerical precision machines prevent exact cancellation of errors $\varepsilon_{k}^{f k m}$ or $\varepsilon_{k}^{i k m}$. An attempt to make them as small as possible is made in the present work to minimize the square of the norm of these errors. This leads to definition of a quadratic criterion $c$ such that $c=\varepsilon^{\mathrm{T}} \varepsilon$ and then to minimization of it. For this, we used a nonlinear least squares based optimization method which usually resolves such multi-dimensional problems. 


\subsection{Jacobian computation}

\subsubsection{Jacobian of FKM method}

The determination of the parameters of the Jacobian $J_{f k m}^{k, P}$ is required to calculate the derivative of the position provided by the FKM w.r.t. the parameters $P$. The implicit equations of IKM gives $i$ for each segment corresponding to each measurement configuration $k$. Now $F_{i k m}^{k, i}=0$. Differentiating the equation for segment $i$ w.r.t. the geometrical parameters, Eq. (3.6), and relative position of the manipulator $\chi_{k}=\left[x_{k}, y_{k}, z_{k}\right]$, we get

$$
\frac{\partial F_{i k m}^{k, i}}{\partial P_{i}} \Delta P_{i}+\frac{\partial F_{i k m}^{k, i}}{\partial \chi_{k}} \Delta \chi_{k}=0
$$

Rewriting in the matrix form

$$
\left[\begin{array}{ccc}
\frac{\partial F_{i k m}^{k, 1}}{\partial P} & 0 & 0 \\
0 & \frac{\partial F_{i k m}^{k, 2}}{\partial P} & 0 \\
0 & 0 & \frac{\partial F_{m g i}^{k, 3}}{\partial P}
\end{array}\right] \Delta P+\left[\begin{array}{c}
\frac{\partial F_{i k m}^{k, 1}}{\partial \chi_{k}} \\
\frac{\partial F_{i k m}^{k, 2}}{\partial \chi_{k}} \\
\frac{\partial F_{i k m}^{k, 3}}{\partial \chi_{k}}
\end{array}\right] \Delta \chi_{k}=0
$$

where

$$
\begin{aligned}
& \Delta P=\left[\Delta P_{1}^{\mathrm{T}}, \Delta P_{2}^{\mathrm{T}}, \Delta P_{3}^{\mathrm{T}}\right]^{\mathrm{T}} \\
& \Delta P_{i}^{\mathrm{T}}=\left[\Delta D x_{i}, \Delta D y_{i}, \Delta D z_{i}, \Delta \theta_{i}, \Delta \alpha_{i}, \Delta L a_{x_{i}}, \Delta L a_{y_{i}}, \Delta L b_{i}\right]
\end{aligned}
$$

Alternatively,

$$
\left[J_{\chi}^{k}\right]_{3 \times 3}\left[\Delta \chi^{k}\right]_{3 \times 1}=\left[J_{P}^{k}\right]_{3 \times 24}\left[\Delta P^{k}\right]_{24 \times 1}
$$

If $J_{\chi}^{k}$ is invertible, we obtain

$$
\Delta \chi^{k}=J_{k, P}^{f k m} \Delta P
$$

where $J_{k, P}^{f k m}=\left(J_{\chi}^{k}\right)^{-1}\left(J_{P}^{k}\right)$.

The Jacobian $J_{P}^{f k m}$ for all errors $\varepsilon_{k}^{f k m}$ with $k=1, \ldots, N_{c}$ is given by

$$
J_{P}^{f k m}=\left[\begin{array}{c}
J_{1, P}^{f k m} \\
\vdots \\
J_{k, P}^{f k m} \\
\vdots \\
J_{N_{c}, P}^{f k m}
\end{array}\right]=\left[\begin{array}{c}
-\left(J_{\chi}^{1}\right)^{-1} J_{P}^{1} \\
\vdots \\
-\left(J_{\chi}^{k}\right)^{-1} J_{P}^{k} \\
\vdots \\
-\left(J_{\chi}^{N_{c}}\right)^{-1} J_{P}^{N_{c}}
\end{array}\right]_{3 \times N_{c} \times 24}
$$

\subsubsection{Jacobian of IKM method}

For the inverse method, a symbolic form of constraint equations $\varepsilon_{i, k}^{i k m}$ is considered. The Jacobian parameter is easier to calculate in the IKM method compared to the FKM method. Since the calibration of each segment is independent, the Jacobian parameters $\varepsilon_{i k m}^{k, P_{i}}$ of the segment $i$ for the configuration $k$ is calculated by differentiating the error $\varepsilon_{k, P_{i}}^{i k m}$ (a single equation) w.r.t. the parameters $P_{i}$, i.e.

$$
J_{k, P i}^{i k m}=\frac{\partial \varepsilon_{i, k}^{i k m}}{\partial P_{i}}
$$


For Model 24, the equation corresponding to the derivative of each error $k=1, \ldots, N_{c}$ for the segment $i$ is given by

$$
J_{k, P i}^{i k m}=\left[\frac{\partial \varepsilon_{i, k}^{i k m}}{\partial D x_{i}}, \frac{\partial \varepsilon_{i, k}^{i k m}}{\partial D y_{i}}, \frac{\partial \varepsilon_{i, k}^{i k m}}{\partial D z_{i}}, \frac{\partial \varepsilon_{i, k}^{i k m}}{\partial \theta_{i}}, \frac{\partial \varepsilon_{i, k}^{i k m}}{\partial \alpha_{i}}, \frac{\partial \varepsilon_{i, k}^{i k m}}{\partial L a x_{i}}, \frac{\partial \varepsilon_{i, k}^{i k m}}{\partial L a y_{i}}, \frac{\partial \varepsilon_{i, k}^{i k m}}{\partial L b_{i}}\right]
$$

The Jacobian corresponding to the derivative of all $N_{c}$ errors $\varepsilon_{i, k}^{i k m}$ for the segment $i$ w.r.t. the parameters $P_{i}$ can be expressed as

$$
J_{k, P_{i}}^{i k m}=\left[\begin{array}{cccccccc}
\frac{\partial \varepsilon_{i, 1}^{i k m}}{\partial D x_{i}} & \frac{\partial \varepsilon_{i, 1}^{i k m}}{\partial D y_{i}} & \frac{\partial \varepsilon_{i, 1}^{i k m}}{\partial D z_{i}} & \frac{\partial \varepsilon_{i, 1}^{i k m}}{\partial \theta_{i}} & \frac{\partial \varepsilon_{i, 1}^{i k m}}{\partial \alpha_{i}} & \frac{\partial \varepsilon_{i, 1}^{i k m}}{\partial L a x_{i}} & \frac{\partial \varepsilon_{i, 1}^{i k m}}{\partial L a y_{i}} & \frac{\partial \varepsilon_{i, 1}^{i k m}}{\partial L b_{i}} \\
\vdots & \vdots & \vdots & \vdots & \vdots & \vdots & \vdots & \vdots \\
\frac{\partial \varepsilon_{i, k}^{i k m}}{\partial D x_{i}} & \frac{\partial \varepsilon_{i, k}^{i k m}}{\partial D y_{i}} & \frac{\partial \varepsilon_{i, k}^{i k m}}{\partial D z_{i}} & \frac{\partial \varepsilon_{i, k}^{i k m}}{\partial \theta_{i}} & \frac{\partial \varepsilon_{i, k}^{i k m}}{\partial \alpha_{i}} & \frac{\partial \varepsilon_{i, k}^{i k m}}{\partial L a x_{i}} & \frac{\partial \varepsilon_{i, k}^{i k m}}{\partial L a y_{i}} & \frac{\partial \varepsilon_{i, k}^{i k m}}{\partial L b_{i}} \\
\vdots & \vdots & \vdots & \vdots & \vdots & \vdots & \vdots & \vdots \\
\frac{\partial \varepsilon_{i, N_{c}}^{i k m}}{\partial D x_{i}} & \frac{\partial \varepsilon_{i, N_{c}}^{i k m}}{\partial D y_{i}} & \frac{\partial \varepsilon_{i, N_{c}}^{i k m}}{\partial D z_{i}} & \frac{\partial \varepsilon_{i, N_{c}}^{i k m}}{\partial \theta_{i}} & \frac{\partial \varepsilon_{i, N_{c}}^{i k m}}{\partial \alpha_{i}} & \frac{\partial \varepsilon_{i, N_{c}}^{i k m}}{\partial L a x_{i}} & \frac{\partial \varepsilon_{i, N_{c}}^{i k m}}{\partial L a y_{i}} & \frac{\partial \varepsilon_{i, N_{c}}^{i k m}}{\partial L b_{i}}
\end{array}\right]_{N_{c} \times 8}
$$

The principle advantage of this method is that the error corresponding to each arm is independent of the errors obtained on other arms of the robot. The calibration process can be decomposed into successive parameters $P^{i}$ for identification of each arm. However, the number of unknown variables are divided by the number of the robot arm (3 for the Delta robot) to simplify the solution.

\section{Simulations}

Several simulations have been carried out by varying the number of parameters to test the effectiveness of the basic methods. For fair comparison, the same measurement configurations for simulation of the two basic methods are considered. These patterns are generated randomly within the workspace of the robot. The inverse kinematics Jacobian associated with these positions is evaluated for any singularity. The resulting equations are generically independent. The simulation is based on an optimization algorithm which requires an initial estimate of the parameters. A uniformly distributed error $P_{r}$ is added with noise to simulate the nominal parameters $P_{r}$. From these parameters, the calibration methods determine the settings to give calibration parameters $P_{c}$. The solution $P_{c}$ is insufficient to assess the validity of the calibration. Therefore, a calibration gain $G$ is defined as

$$
G=100 \cdot \frac{\left\|P_{r}-P_{n}\right\|-\left\|P_{r}-P_{c}\right\|}{\left\|P_{r}-P_{n}\right\|}
$$

The calibration gain $G$ closer to $100 \%$ indicates a great improvement of parameter estimation, which means that the method converges to the exact solution, i.e. errors obtained (norm of the difference between the parameters estimated by calibration and the actual parameters of the robot) are negligible.

\subsection{Simulations without measurement noise}

First, the simulation is carried out without considering noise in the measurements. The initial estimate of the error in the parameters is one millimetre. The results from 8 measurement configurations demonstrate that the calibration gains are closed to $G=100 \%$ for both methods. 
The simulation time using the forward method and the inverse method is, respectively, $t=$ $0.911 \mathrm{~s}$ and $t=2.906 \mathrm{~s}$. For errors on initial estimates of one centimetre, the forward method does not converge at all, and thus the resulting solution is insignificant. This results in a large numerical instability of this calibration method. On the other hand, the inverse method converges successfully. The simulation time using the forward method and the inverse method is $t=1.395 \mathrm{~s}$ and $t=3.385 \mathrm{~s}$, respectively.

\subsection{Simulations with measurement noise}

\subsubsection{FKM}

First, we added a noise of one micron in the measurements of the position of the end effector and length and a noise of one hundredth of a degree in the joint angles corresponding to the calibration simulation for 8 measurement configurations. For an error of one tenth of a millimeter, a negative gain is obtained, which means we cannot improve the knowledge of geometric parameters of the robot ( $G=-38.54 \%$ is the percentage average of the gain on all parameters). We can improve results by increasing the number of measurement configurations. By doing the same simulation but with 20,40 and 60 configurations, the percentage average of the gain on all parameters of $20 \%, 38 \%, 45.38 \%$ is obtained with the simulation time of $4.256 \mathrm{~s}, 5.247 \mathrm{~s}$ and $10.945 \mathrm{~s}$, respectively, thus improving the results. For errors higher than $0.1 \mathrm{~mm}$, the forward method provides unsatisfactory results (negative calibration gain of $G=-260 \%$ with $30.877 \mathrm{~s}$ ). The major problem of the forward method is that for a number of parameters to be determined (24 in our example), the optimization algorithms hardly or even do not converge. Thus, this method is unreliable and slow. Figure 2 shows an example of the simulation results using FKM where the number of measurement configurations is equal to 60 and the estimated error on the parameters is $0.01 \mathrm{~mm}$.

(a)

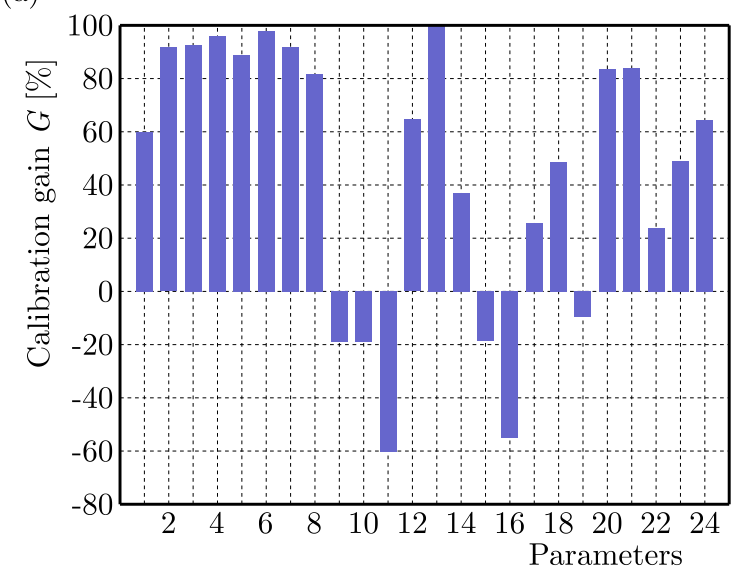

(b)

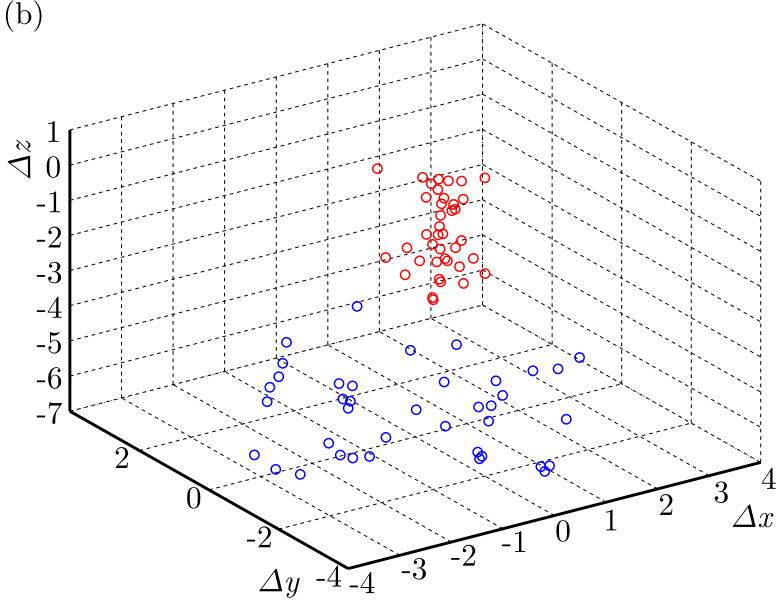

Fig. 2. Simulation result based on FKM: (a) gain of calibration of each parameter, (b) evaluation test of the forward method using the error in the operating points before (blue points) and after (red points) the calibration

\subsection{2. $I K M$}

Based on the simulated method without considering the measurement error, we can determine exactly the geometric parameters of the robot with a number of configuration $N c>7$. For relatively low measurement errors $(0.001 \mathrm{~mm}$ and 0.001 degrees $)$ and errors on large estimates (about a centimeter), the results show that the parameters are significantly improved (calibration gain close to $100 \%$ ). For errors on the initial estimate of one centimeter (the error here is 
ten times higher compared with FKM), the convergence of the inverse method is observed with $G=99.9 \%$ in $3.385 \mathrm{~s}$. The results obtained using the Levenberg-Marquardt and Gauss-Newton approaches are similar. For measurement errors in the order of $1 \mathrm{~mm}$ and 0.01 degree on the parameters, the results show that 8 configurations are not enough to improve the parameters estimation where the calibration gain obtained is $G=-6.17 \%$ in 2.578 s. It is therefore necessary to increase the numbers of measurement configurations to 20,40 and 60 , with the resulting percentage average of the gain on all parameters found as $62.54 \%, 87.58 \%, 86.5 \%$ in simulation times of $5.781 \mathrm{~s}, 8.877 \mathrm{~s}$ and $11.907 \mathrm{~s}$, respectively. Comparing the inverse method with the forward one, it is found that the former is more robust to measurement errors and is faster and has a greater radius of convergence. Figure 3 shows an example of the simulation results using IKM where the number of measurement configurations is 60 and the estimated error on the parameters is equal to $1 \mathrm{~mm}$.

(a)

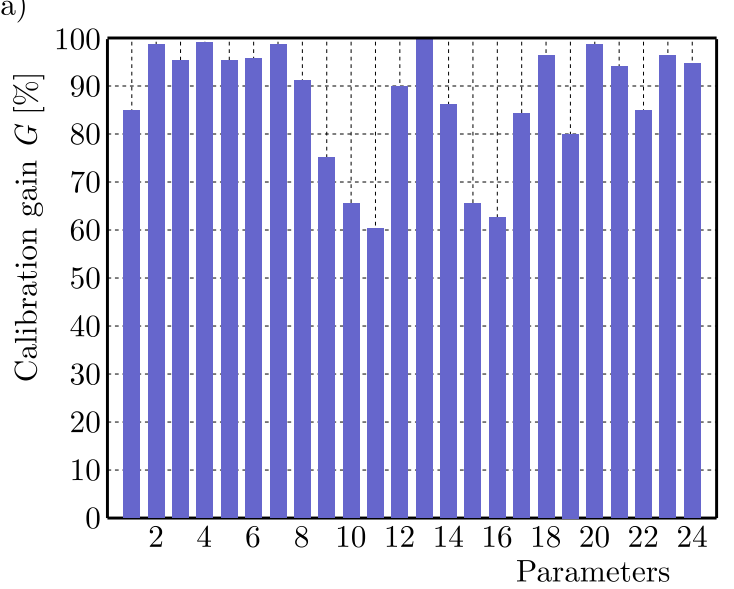

(b)

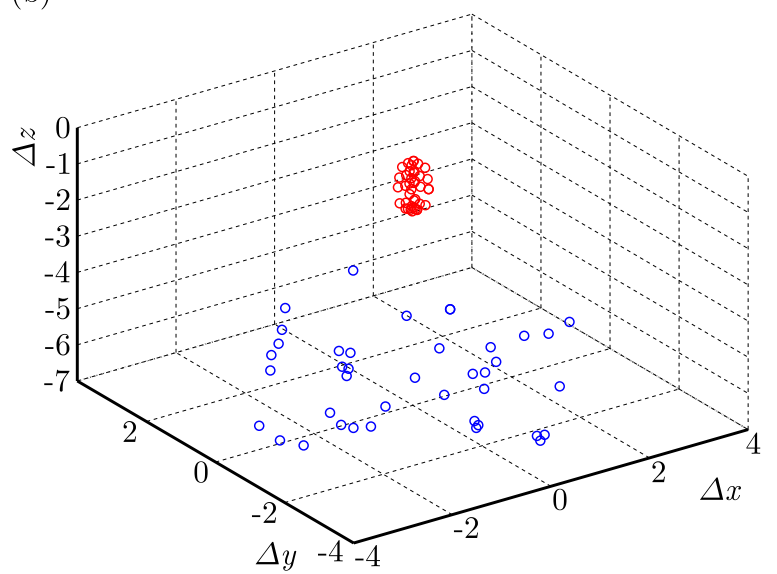

Fig. 3. Simulation result based on IKM: (a) gain of calibration of each parameter, (b) evaluation test of the inverse method using the error in the operating points before (blue points) and after (red points) the calibration

\section{Discussion}

In this study, we adapted traditional methods used for serial robots on Delta parallel robot. The simulation allowed us to highlight the benefit of the inverse method compared to the forward method. In the inverse method, the calibration gains near $100 \%$ are achieved thereby demonstrating significant improvement of the parameters until convergence to the exact solution. However, in comparison with the forward method, the gain observed in this case is $48.27 \%$. The subdivisions of the calibration parameter identification chain by chain of the joints and the form of the equations of formal constraints improve the algorithm convergence speed and its robustness. The simulation results also highlighted challenges faced by the numerical calibration methods. This is primarily due to convergence problems in optimization methods, which provide a solution that minimizes the criterion but does not achieve the desired outcome. The key reason behind this challenge is measurement noise that modifies the equations of constraints. The resulting solution considers an error function of measurement noise, types of methods used and constraints, and the number and quality of measurement configurations. If this error has the same order of magnitude as the initial estimated optimization procedures, we cannot guarantee the quality of calibration. To deal with the number of parameters to be taken into account and to improve the accuracy of results, we have to increase the number of measurement configurations. However, we have observed the limits where the results do not improve more 
than 50 considered positions (see Fig. 4). Other possible strategy involves taking into account the distribution of noise in the measurements and to choose the measurement configurations. The proposed solution seems to offer very interesting perspectives. It is therefore not necessary to consider further measurement configurations.

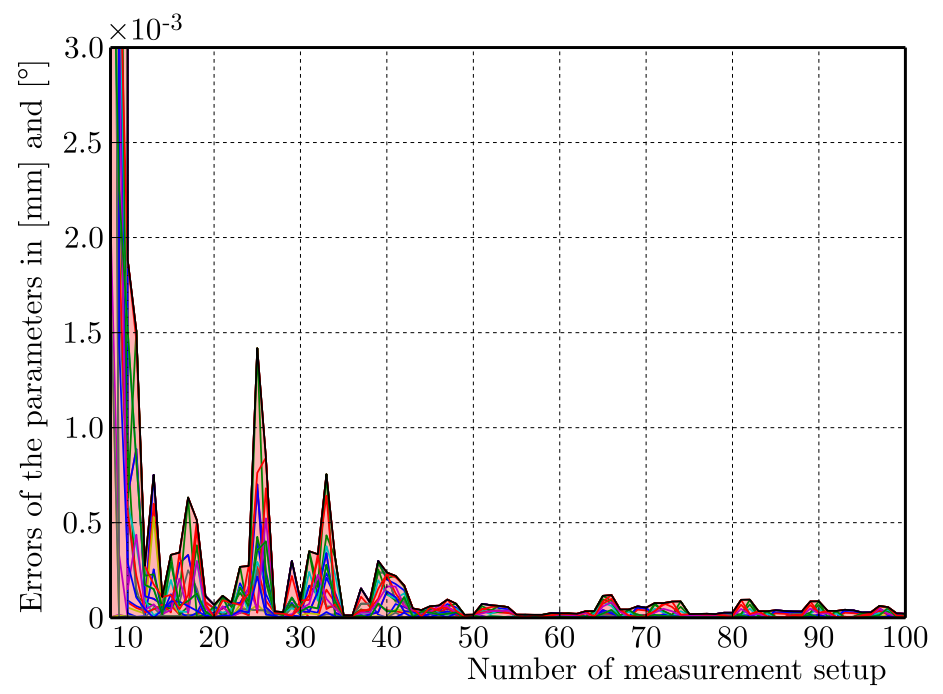

Fig. 4. Influence of measurement configuration on the results of the inverse method in the presence of errors on the parameters of $0.1 \mathrm{~mm} /$ degrees for lengths/angles and with amplitude noise position measurements equal to $1 \mu \mathrm{m}$

\section{Conclusion}

In this research, the calibration methods used traditionally for serial robots have been proposed for parallel robots. Considering an example of 'Delta' robot, the simulation results highlighted over-performance of the inverse method compared to the forward method. The proposed subdivision concept of the calibration parameters (i.e. segment by segment identification) and the constraints of the inverse method equations improve the convergence of the algorithm toward the optimal solution. The future work can be related with going beyond the optimization procedures and focusing on the adaptation tools with distributed measurement errors taken into consideration.

\section{References}

1. Ajwad S.A., Iqbal J., Islam R.U., Alsheikhy A., Almeshal A., Mehmood A., 2018, Optimal and robust control of multi DOF robotic manipulator: Design and hardware realization, Cybernetics and Systems, 49, 1, 77-93

2. Alam W., Mehmood A., Ali K., Javaid U., Alharbi S., Iqbal, J., 2018, Nonlinear control of a flexible joint robotic manipulator with experimental validation, Strojniski vestnik - Journal of Mechanical Engineering, 64, 1, 47-55

3. Axehill J.W., Dressler I., Gunnarsson S., Robertsson A., NorrlöF M., 2014, Estimation-based (ILC) applied to a parallel kinematic robot, Control Engineering Practice, 33, $1-9$

4. Bai P., Jiangping Mei J., Huang T., Chetwynd D.G., 2015, Kinematic calibration of Delta robot using distance measurements, Proceedings of the Institution of Mechanical Engineers, Part C: Journal of Mechanical Engineering Science, 230, 3, 414-424 
5. Bentaleb T., Shahin L., 2015, Humanoid robots imitation of human motion using offline and real-time adaptation techniques, International Journal of Mechanisms and Robotic Systems, 2, 3/4, 295-313

6. Bentaleb T., Zennadi K., Belouchrani M.E.A., 2007, A new method for self-calibration of the parallel robot "DELTA" using a constrained link, 18th French Congress of French Mechanics, Grenoble, France, 1-6

7. Besnard S., Khalil W., 2001, Identifiable parameters for parallel robots kinematic calibration, IEEE International Conference on Robotics and Automation, 3, 2859-2866

8. FAn C., Zhao G., Zhao J., Zhang L., Sun L., 2015, Calibration of a parallel mechanism in a serial-parallel polishing machine tool based on genetic algorithm, International Journal of Advanced Manufacturing Technology, 81, 27-37

9. Ibrahim O., Khalil W., 2010, Inverse and direct dynamic models of hybrid robots, Mechanism and Machine Theory. 45, 4, 627-640

10. Iqbal J., Ajwad S.A., Abbas S.Z., Khan A.A., Islam R.U., 2016, Automating industrial tasks through mechatronic systems - A review of robotics in industrial perspective, Technicki VjesnikTechnical Gazette, 23, 3, 917-924

11. Kamali K., Joubair A., Bonev I.A., Bigras P., 2016, Elasto-geometrical calibration of an industrial robot under multidirectional external loads using a laser tracker, IEEE International Conference on Robotics and Automation, Stockholm, Sweden, 4320-4327

12. Mei J., Ni Y., Li Y., Zhang L., Liu F., 2009, The error modeling and accuracy synthesis of a 3-DOF parallel robot delta-S, International Conference on Information and Automation, Zhuhai, Macau, 289-294

13. Meng Y., Zhuang H., 2007, Autonomous robot calibration using vision technology, Robotics and Computer-Integrated Manufacturing, 23, 4, 436-446

14. Merlet J.-P., 2006, Parallel robots, Solid Mechanics and Its Applications, Springer, 128, ISBN: 978-1-4020-4132-7

15. Mo J.S., QIU C., Zeng L., Zhang X.M., 2016, A new calibration method for a directly driven 3PRR positioning system, Journal of Intelligent $\&$ Robotic Systems, 85, 3-4, 613-631

16. NGuyen H.N., Zhou J., KANG H.K., 2015, A calibration method for enhancing robot accuracy through integration of an extended Kalman filter algorithm and an artificial neural network, Neurocomputing, 151, 996-1005

17. Nubiola A., Bonev I.A., 2014, Absolute robot calibration with a single telescoping ballbar, Precision Engineering, 38, 472-480

18. Pagis G., Bouton N., Briot S., Martinet P., 2015, Enlarging parallel robot workspace through Type-2 singularity crossing, Control Engineering Practice, 39, 1-11

19. RAUF A., RYu J., 2001, Fully autonomous calibration of parallel manipulators by imposing position constraint, IEEE International Conference on Robotics and Automation, Proceedings, 3, 2389-2394

20. Viera P., Juraj U., Vladimr B., Peter S., 2011, Delta Robots - Robots for high speed manipulation, Technicki Vjesnik - Technical Gazette, 18, 3, 435-445

21. YU D.Y., 2008, Parallel robots pose accuracy compensation using artificial neural networks, IEEE International Conference on Mechatronics and Automation, Takamatsu, 750-754

22. Yu D.Y., Cong D.C., Han J.W., 2005, Parallel robots pose accuracy compensation using artificial neural networks, International Conference on Machine Learning and Cybernetics, 5, 3194-3198

23. Zhao L., Joubair A., Bigras P., Bonev I.A., 2015, Metrological evaluation of a novel medical robot and its kinematic calibration, International Journal of Advanced Robotic Systems, 12, 9, 1-13 